1. Harper, J. W. \& Elledge, S. J. Curr. Opin. Genet. Dev. 6, 56-64 (1996).

2. Ficlds, S. \& Song, O.-K. Nature 340, 245-246 (1989).

3. Polyak, K. et al. Cell 78, 59-66 (1994).

4. Toyoshima, H. \& Hunter, T. Cell 78, 67-74 (1994).

5. Ferreira, P. C. G., Hemerly, A. S., Villarroel, R., Van Montagu, M. \& Inzé, D. Plant Cell 3, 531-540 (1991).

6. Hirahama, T., Imajuku, Y., Anai, T., Matsui, M. \& Oka, A. Gene 105, 159-165 (1991).

7. Martinez, M. C., Jørgensen, J.-E., Lawton, M. A., Lamb, C. J. \& Doerner, P. W. Proc. Natl Acad. Sci. USA 89, 7360-7364 (1992).

8. Hemerly, A. et al. EMBO J. 14, 3925-3936 (1995)

9. Harper, J. W., Adami, G. R., Wei, N., Keyomarsi, K. \&

Elledge, S. J. Cell 75, 805-816 (1993).

10. Grafi, G. \& Larkins, B. A. Science 269, 1262-1264 (1995).

\section{Determining cortical}

\section{landscapes}

The folded surface of the cerebral cortex contains a mosaic of functionally distinct areas, connected by a network of nerve fibres in the sub-cortical white matter. This network determines, in part, the computations that the cortex performs. The folds help to fit the large sheet-like cortex into a compact space ${ }^{1}$, but this does not explain the species specificity of their pattern, or their characteristic relationship with the locations of the cortical areas ${ }^{2}$. Here I present data in support of
Van Essen's Hypothesis ${ }^{2}$ to explain these features.

Local wiring - preferential connectivity between nearby areas of the cortical sheet - is a simple strategy that helps keep cortical connections short ${ }^{3-5}$. In principle, efficient cortical folding could further reduce connection length, in turn reducing white matter volume and conduction delays $^{2,6}$. Such an arrangement should match convex folds (gyri) with groups of heavily connected areas, and concavities (sulci) with sparsely connected regions. Van Essen $^{2}$ has suggested that competing mechanical tension in the axons of the developing cortical network produces such efficient folding.

Cats $^{5}$ and macaques ${ }^{7}$ have roughly 70 cortical areas in each folded hemisphere, linked by around 1,000 connections. The complexity makes it possible to find subsets of connections to support virtually any theory of cortical organization. Therefore, I analysed collated anatomical data quantitatively to see if the 3-dimensional folding of the cortex is in fact related to wiring. I examined the folding and connections between areas that are 'nearest neighbours' or 'next-door-but-one $e^{3-5}$ on the sheet. In both animals, connectivity was significantly denser between nearby areas on the same gyrus than between nearby areas sep-

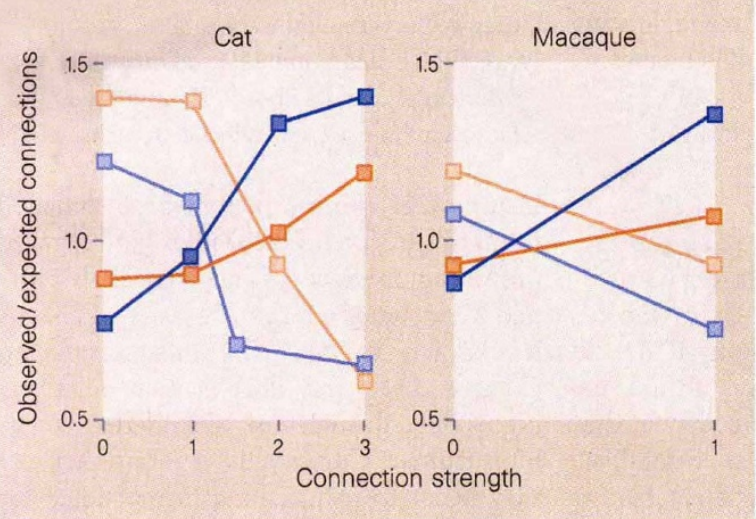

Figure 1 Local connections are denser within gyri (dark blue and orange) than across sulci (pale blue and orange). Nearest neighbours plus next-door-butone areas (blue) and neighbouring areas alone (orange) are compared. The ratio of observed/expected connections is plotted against connection weight. The differences between observed and expected connections are significant (see Table 1).

\begin{tabular}{|c|c|c|c|c|c|c|c|}
\hline \multirow{2}{*}{\multicolumn{2}{|c|}{ Weight }} & \multicolumn{2}{|c|}{ (1) NN and NDB1 } & \multicolumn{2}{|c|}{ (2) $N N$} & \multicolumn{2}{|c|}{ (3) All } \\
\hline & & Gyri & Sulci & Gyri & Sulci & $\mathrm{NN}$ and NDB1 & Non-local \\
\hline \multirow[t]{5}{*}{ Cat } & 0 & $105 / 138$ & $172 / 139$ & $31 / 37$ & $23 / 17$ & $277 / 452$ & $1,515 / 1,338$ \\
\hline & 1 & $56 / 63$ & $70 / 63$ & $16 / 19$ & $12 / 9$ & $126 / 99$ & $266 / 293$ \\
\hline & 2 & $122 / 94$ & $66 / 94$ & $71 / 67$ & $27 / 31$ & $188 / 85$ & $147 / 250$ \\
\hline & 3 & $47 / 35$ & $24 / 36$ & $34 / 29$ & $8 / 13$ & $71 / 23$ & $23 / 70$ \\
\hline & & \multicolumn{2}{|c|}{$P<0.0001$} & \multicolumn{2}{|c|}{$P<0.04$} & \multicolumn{2}{|c|}{$P<0.0001$} \\
\hline \multirow[t]{2}{*}{ Macaque } & 0 & $211 / 255$ & $344 / 300$ & $70 / 78$ & $51 / 43$ & $556 / 751$ & $3,498 / 3,301$ \\
\hline & 1 & $195 / 151$ & $132 / 176$ & $122 / 114$ & $53 / 61$ & $327 / 130$ & $378 / 574$ \\
\hline
\end{tabular}

The cortex shows efficient folding and local wiring. Number of observed (bold) over number of expected connections are compared. Comparisons are (1) within gyri compared with across sulci connections between nearest neighbour (NN) and next door but one (NDB1) areas, (2) within gyri compared with across sulci connections between NN areas, (3) all NN and NDB1 compared with connections between all non-local areas ${ }^{3-5}$. Weight gives the ordinal rank of the strength of connection. Cat ${ }^{5}$ connection density ran from ' 0 ' (no connection) to ' 3 ' (strong connection). Macaque connections were either ' 0 ' (absent) or ' 1 ' (present). Probabilities were calculated using $\chi^{2}$. If local wiring and efficient folding were absent, the observed values in the table would equal the expected values, hence the table shows that, for NN and NDB1 areas, local wiring contributes more than folding to efficient cortical connectivity. Full details of anatomical data are available at http://www.psychology.ncl.ac.uk/jack/gyri.html.

arated by sulci (Fig. 1, Table 1).

In the cat, where data are available on the relative strengths of connections, neighbouring and next-door-but-one areas within gyri had more moderate and strong connections whereas those separated by sulci had more sparse connections $(P<0.05$ by binomial tests). Thus the development of the cortex does seem to coordinate folding with connectivity in a way that produces smaller and faster brains. Many mechanisms could conceivably account for this (for example, growth factors from extrinsic connections), but the result is quantitatively consistent with Van Essen's theory of morphogenesis ${ }^{2}$.

Despite the presence of local wiring ${ }^{3,5}$ and efficient folding ${ }^{2,6}, 53 \%$ of connections in the cat link areas that are neither neighbours nor next-door-but-one, whereas $42 \%$ of such areas remain unconnected (Table 1). Furthermore, $42 \%$ of the connections between nearest neighbours and next-door-but-one areas cross sulci, whereas $32 \%$ of such areas within gyri are unconnected. Similar figures apply to the macaque (Table 1). Thus natural selection has produced a 3-dimensional arrangement of areas that is not optimal for minimizing wiring. Indeed, the connectional architecture could be perfectly accomodated only if local wiring and folding were able to take place in more than three dimensions $s^{5,7,8}$. Brain design represents a compromise between efficient wiring and other factors ${ }^{8}$.

Imagine a cortex in which it were possible to shuffle the areas and folds while keeping connections the same. Shuffling the areas (abolishing local wiring) would compromise compact wiring more than shuffling the folds (abolishing efficient folding; Table 1). Morphologically spectacular folding may, thus, 'tune up' a cortex that is already reasonably compact from local wiring, particularly in larger brains where wiring 'costs' are greater ${ }^{1,9}$. I anticipate, however, that local wiring rules apply even in those cortices that do not fold.

Jack W. Scannell

Neural Systems Group,

Psychology Department,

University of Newcastle,

Newcastle-upon-Tyne NEI 7RU, UK

e-mail: j.w.scannell@ncl.ac.uk

Welker, W. in Cerebral Cortex Vol. 8B (eds Jones, E. G. \& Peters, A.) 3-136 (Plenum, New York, 1990).

2. Van Essen, D. C. Nature 385, 313-318 (1997)

3. Young, M. P. Nature 358, 152-155 (1992)

4. Cherniak, C. J. Neurosci. 14, 2418-2427 (1994).

5. Scannell, J. W., Young, M. P. \& Blakemore C. J. Neurosci. 15, 1463-1483 (1995)

6. Griffin, L. J. Theoret. Biol. 166, 261-273 (1994).

7. Young, M. P. Proc. R. Soc. Lond. B 252, 13-18 (1993).

8. Young, M. P. \& Scannell J. W. Trends Neurosci. 19,413-415 (1996).

9. Ringo, J. L. Brain Behav. Evol, 38, 1-16 (1991). 